\title{
Health status in patients with COPD treated with roflumilast: two large noninterventional real-life studies: DINO and DACOTA
}

This article was published in the following Dove Press journal: International Journal of COPD

\author{
Peter Kardos' \\ Ingo Mokros ${ }^{2}$ \\ Rüdiger Sauer ${ }^{3,4}$ \\ Claus F Vogelmeier ${ }^{5,6}$ \\ 'Group Practice and Centre for \\ Allergy, Respiratory, and Sleep \\ Medicine, at Maingau Red Cross \\ Hospital, Frankfurt am Main, \\ Germany; ${ }^{2}$ AstraZeneca GmbH \\ Unternehmensbereich Medizin, \\ Wedel, Germany; ${ }^{3}$ Group Practice \\ with Respiratory, Allergy and Sleep \\ Unite, Lung Center, Ulm, Germany; \\ ${ }^{4}$ Health Center Clinic Blaubeuren, \\ Blaubeuren, Germany; ${ }^{5}$ Department \\ of Medicine, Pulmonary and Critical \\ Care Medicine, University Medical \\ Center Giessen and Marburg, \\ Philipps-University Marburg, Marburg, \\ Germany; ${ }^{6}$ German Center for Lung \\ Research (DZL), Giessen, Germany
}

Correspondence: Peter Kardos Group Practice and Centre for Allergy, Respiratory, and Sleep Medicine, at Maingau Red Cross Hospital, Scheffelstrasse 33, D-603 I 8 Frankfurt am Main, Germany

Tel +49695536I I

Email kardos@lungenpraxis-maingau.de
Purpose: DINO and DACOTA were prospective, noninterventional studies assessing the health status and quality of life of patients with COPD newly treated with roflumilast $500 \mu \mathrm{g}$ once-daily add-on therapy.

Patients and methods: Patients were evaluated over 6 months. Clinical COPD questionnaire (CCQ) and COPD assessment test (CAT) scores were recorded at baseline and after 3 and 6 months. In DACOTA, post-bronchodilator $\mathrm{FEV}_{1}$ was recorded at each time point.

Results: Of 5,462 and 3,645 patients recruited into DINO and DACOTA, respectively, 3,274 patients in DINO and 916 patients in DACOTA completed the 6-month visit. Almost all patients had severe or very severe airway obstruction; mean baseline CCQ total score was 3.9 in DINO and 3.7 in DACOTA. Overall, $33.8 \%$ of patients in DACOTA and $30.6 \%$ in DINO discontinued treatment prematurely. Significant and clinically relevant improvements in CCQ total scores were observed in both studies (mean change from baseline of 1.36 in DINO and 0.91 in DACOTA at Month 6 [all $P<0.001]$ ). Changes in CAT total score from baseline to Month 6 indicated that the average clinical impact of COPD was reduced from a severe (score: $21-30$ ) to a moderate (score: 11-20) impairment. In DACOTA, mean change in post-bronchodilator $\mathrm{FEV}_{1}$ was $202 \mathrm{~mL}(P<0.001)$. Diarrhea, nausea, and weight decrease were the most frequently reported adverse drug reactions.

Conclusion: In real-life clinical practice, roflumilast treatment as an add-on therapy is associated with clinically relevant improvements in health status and quality of life.

Keywords: quality of life, phosphodiesterase-4 inhibitor, lung function, observational study, roflumilast, clinical COPD questionnaire, COPD assessment test

\section{Introduction}

Many patients with COPD continue to suffer exacerbations and recurrent hospitalizations despite treatment with long-acting muscarinic antagonists (LAMA), long-acting beta-agonists (LABA), and inhaled corticosteroids (ICS). Roflumilast is a selective phosphodiesterase-4 (PDE4) inhibitor recommended for maintenance treatment of $\mathrm{COPD}$ in patients with post-bronchodilator $\mathrm{FEV}_{1}<50 \%$ predicted, associated with chronic bronchitis and a history of exacerbations, as add-on to inhaled therapies. ${ }^{1}$ Randomized controlled trials (RCTs) have demonstrated that roflumilast effectively reduces exacerbations and hospitalizations when added on top of inhaled combination therapy in patients with severe COPD and a history of exacerbations. ${ }^{2-5}$ While the primary effect of roflumilast is to reduce the risk of exacerbations, it has also recently been shown to reduce hyperinflation and elicit a significant improvement in $\mathrm{FEV}_{1} \cdot{ }^{6}$ 
RCTs are considered the gold standard of study design, but often exclude large populations of patients, such as the elderly, those with mild or very severe disease, or patients with comorbid conditions that pose a risk of adverse events. ${ }^{\text {? }}$ Therefore, these study populations may not be representative of those typically encountered in daily practice. ${ }^{7}$ By contrast, real-life observational studies are conducted in broad patient populations, with a range of demographics, disease characteristics, and comorbidities. ${ }^{7,8}$ Treatment adherence is usually much higher and physician visits are more frequent in RCTs than in real life. Also, RCTs often assess surrogate endpoints (eg, $\mathrm{FEV}_{1}$ ), which do not directly reflect patients' quality of life (QoL). Indeed, results from a Cochrane review of 34 RCTs that evaluated the efficacy and safety of two oral PDE4 inhibitors, roflumilast (20 trials with 17,627 participants) and cilomilast (14 trials with 6,457 participants), reported that while both agents provided benefit over placebo in improving lung function and reducing the likelihood of exacerbations, they had little impact on QoL or symptoms. ${ }^{9}$ Consequently, while RCTs are important to assess efficacy, observational trials, particularly those with an emphasis on patient-reported outcomes, are needed to assess the effectiveness of therapies outside the controlled setting.

QoL measures are important for evaluating treatments prescribed to patients, and it can be argued that they are best assessed in the real-life setting. Studies evaluating QoL in the real-life setting are currently lacking for roflumilast.

To document the effects of roflumilast in daily clinical practice, two noninterventional studies were conducted in Germany: DINO (NCT01285180) and DACOTA (NCT01285167). The primary objective of both the studies was to monitor the effects of roflumilast on health status in patients with severe/very severe COPD prescribed this agent for the first time as an add-on therapy, over a 6-month period.

\section{Patients and methods Patients and study design}

DINO and DACOTA were pragmatic Phase IV studies conducted in primary and secondary care, respectively.

The design and conduct of DINO and DACOTA are summarized in Table 1. Patients with severe/very severe COPD were eligible for inclusion if they fulfilled the criteria for receiving roflumilast treatment as per the local label. ${ }^{10}$ For the purpose of enrolment in the studies, the label requirement for a history of frequent exacerbations was defined as $\geq 1$ exacerbations during the previous 12 months. This label was based on clinical studies, which included patients with $\geq 1$ exacerbations in the previous year, with LABA as concomitant medication.

In both studies, COPD severity was classified according to the Global Initiative for Chronic Obstructive Lung Disease (GOLD) 2011 criteria using data derived from patients' medical records; COPD severity was also verified by lung function measurements in DACOTA, but not in DINO. Treatment-naïve patients and patients receiving COPD maintenance therapy were eligible, but patients were not to have received roflumilast prior to the start of the studies. No further inclusion or exclusion criteria were specified; the only restrictions on comorbid conditions or concomitant medications were those detailed in the roflumilast (Daxas) Summary of Product Characteristics. ${ }^{10}$

Table I Comparison of DINO and DACOTA studies

\begin{tabular}{|l|l|l|}
\hline & DINO & DACOTA \\
\hline Study design & Prospective, noninterventional, observational, multicenter & Prospective, noninterventional, observational, multicenter \\
\hline Study sites & 2,195 primary care centers in Germany & 739 secondary care centers in Germany \\
\hline Study period & August 20I0-August 20I2 & January 20II-February 20I2 \\
\hline $\begin{array}{l}\text { Number of } \\
\text { participants }\end{array}$ & $\begin{array}{l}\text { Treated set: 5,375 } \\
\text { Effectiveness set: 3,274 }\end{array}$ & $\begin{array}{l}\text { Treated set: } 3,597 \\
\text { Effectiveness set: } 916\end{array}$ \\
\hline $\begin{array}{l}\text { Eligible } \\
\text { participants }\end{array}$ & $\begin{array}{l}\text { Diagnosis of severe to very severe COPD classified } \\
\text { according to the GOLD criteria using data from patients' } \\
\text { medical records }\end{array}$ & $\begin{array}{l}\text { Diagnosis of severe to very severe COPD classified } \\
\text { according to the GOLD criteria using data from patients' } \\
\text { medical records and verified by lung function measurements }\end{array}$ \\
\hline Treatment & Roflumilast $500 \mu$ g/day for 6 months & Roflumilast $500 \mu$ gg/day for 6 months \\
\hline Primary outcome & Change from baseline in CCQ total score at study end & Change from baseline in CCQ total score at study end \\
\hline $\begin{array}{l}\text { Key secondary } \\
\text { outcomes }\end{array}$ & $\bullet$ Evaluation of health status measured by CAT & $\begin{array}{l}\text { Evaluation of health status measured by CAT } \\
\text { - Spirometry measurements to assess lung function }\end{array}$ \\
\hline
\end{tabular}

Notes: Treated set: all patients who received at least one dose of roflumilast; effectiveness set: a population that was more compliant with the label and the study protocol and had complete data available for the assessment of the primary endpoint; the effectiveness set included all patients in the treated set who met a number of additional criteria. Full details of the effectiveness set criteria can be found in the Supplementary material.

Abbreviations: CAT, COPD assessment test; CCQ, clinical COPD questionnaire; GOLD, Global Initiative for Chronic Obstructive Lung Disease. 
Roflumilast $500 \mu \mathrm{g}$ once daily (OD) was prescribed at the sole discretion of the treating physician. Patient adherence was not monitored. Data were collected at baseline (study entry), and at $\sim 3$ and 6 months after initiation of roflumilast treatment.

Both studies were conducted in accordance with relevant guidelines, including applicable German regulations ( $\$ 67$ Section 6 German Drug Law), the German Federal Institute for Drugs and Medical Devices (BfArM) publication on noninterventional studies, the German Association of Research-Based Pharmaceutical Companies (Verband der Forschenden Arzneimittelhersteller) recommendations, the Declaration of Helsinki, ${ }^{11}$ and Good Pharmacoepidemiology Practices. ${ }^{12}$ For both studies, the protocols and supporting documents were approved by the relevant local ethics committee (Ethikkommission der Landesärztekammer Baden-Württemberg) prior to enrolment. In agreement with local regulations, the physician gave the patient oral and written information about the study, and obtained the patient's voluntary consent for the collection of personal data and transfer of the data in pseudonymous form before the start of the study. All patients provided written informed consent. The patient had the right to withdraw his/her consent at any time.

\section{Assessments}

The primary endpoint was defined as the change from baseline in clinical COPD questionnaire (CCQ) total score after 6 months of treatment with roflumilast. Secondary endpoints included changes from baseline in CCQ domain scores, COPD assessment test (CAT) total score, and postbronchodilator $\mathrm{FEV}_{1}$ (DACOTA only). An analysis of change in CCQ total and domain scores and in CAT total score in frequent versus nonfrequent exacerbator subgroups was performed post hoc.

Health status and QoL were measured using the validated German versions of $\mathrm{CCQ}^{13}$ and $\mathrm{CAT}^{14}$ at baseline, and at Months 3 and 6 of the study. The CCQ consists of 10 items divided into three domains: symptoms, functional state, and mental state. Each item is rated on a 7-point Likert scale, where $0=$ asymptomatic/no limitations and $6=$ extremely symptomatic/totally limited. The CCQ total score is calculated as the mean of the sum of all items. ${ }^{13}$ The minimal clinically important difference (MCID) of the CCQ total score is $0.41 .{ }^{15}$ The CAT consists of eight items (cough, phlegm, chest tightness, breathlessness while going up hills/ stairs, activity limitation at home, confidence leaving home, sleep, and energy) rated on a 6-point scale, where $0=$ no impact and $5=$ very severe impact. ${ }^{14}$ The total CAT score is calculated as the sum of responses given in the eight items with a range of $0-40$; a change of 2 points is considered clinically significant. ${ }^{16}$

Lung function was assessed at all three visits by spirometry in DACOTA. Reversibility of airway obstruction was assessed by spirometry prior to, and 15-30 minutes after bronchodilation using short-acting inhalation aerosols, predominantly salbutamol. The following variables were documented: $\mathrm{FEV}_{1}$, vital capacity $(\mathrm{VC}), \mathrm{FEV}_{1} / \mathrm{VC}, \mathrm{FVC}$, and $\mathrm{FEV}_{1} / \mathrm{FVC}$. DINO focused on the usual care evaluation of patients' health status; therefore, lung function was not assessed.

Safety and tolerability were assessed by physicianreported adverse drug reactions (ADRs), and all data were collected by the treating physician using paper case report forms.

\section{Statistical analyses}

Two populations were defined for analysis: the treated set and the effectiveness set. The treated set comprised all patients who received at least one dose of roflumilast. The effectiveness set comprised all patients in the treated set who had complete data available for the assessment of the primary endpoint, and were more compliant with the label and study protocol. Full details of the criteria for the effectiveness set can be found in the Supplementary material.

All baseline characteristics, safety, and tolerability data are presented for the treated sets; all other data presented herein are for the effectiveness sets, with data for the treated sets detailed in the Supplementary material.

Changes from baseline in the CCQ total and domain scores were analyzed by means of an ANOVA model including the baseline value as an independent variable. Analogous ANOVA models were used to evaluate the changes from baseline in CAT total score and spirometric variables.

Post-hoc subgroup analyses were performed for primary and secondary CCQ endpoints, and for CAT total scores according to the number of moderate exacerbations (ie, those not leading to hospitalization or death, but treated with ICS and/or antibiotics) or severe exacerbations (ie, those leading to hospitalization or death) in the 12 months prior to starting roflumilast treatment. For the purpose of these subgroup analyses, patients were categorized as frequent exacerbators if they had experienced $\geq 2$ moderate or severe exacerbations in the previous year. Exacerbation group comparisons were analyzed by the Wilcoxon rank sum test.

Missing data were not imputed; however, missing data were displayed in patient data listings and were included in 
frequency tables as appropriate. In the event of missing dates, questionnaire scores, and spirometric variables, imputation rules relative to the parameter were applied. The chosen level of significance for statistical testing was 0.05 . Formal sample size calculations were not performed; the sample size was determined on grounds of feasibility.

\section{Results}

\section{Patient demographics and baseline characteristics}

A total of 5,462 patients were enrolled in DINO and 3,645 in DACOTA, of whom 5,375 and 3,597 patients received at least one dose of roflumilast, respectively. The effectiveness sets comprised 3,274 patients in DINO and 916 in DACOTA ( $60.9 \%$ and $25.5 \%$ of the treated sets, respectively). Approximately one-third of patients in the treated set of both studies discontinued prematurely $(30.6 \%$ of the treated set in DINO and $33.8 \%$ in DACOTA). The flow of patients through each study is shown in Figure S1.

Patient demographics and baseline characteristics for the treated sets are shown in Table 2 (effectiveness set data are shown in Table S1). Patients with severe COPD comprised the highest proportion of all patients in both studies; $4.3 \%$ of patients in DINO and $10.1 \%$ in DACOTA had mild or moderate disease. COPD severity was unknown according to the classification described in $1.8 \%$ and $48.6 \%$ of the treated sets in DINO and DACOTA, respectively. Other baseline characteristics were similar between the studies.

\section{Health status}

\section{CCQ and CAT scores}

In the effectiveness sets of both studies, the mean CCQ total score significantly decreased (improved) from baseline to Months 3 and $6(P<0.001$; Figure 1). Significant improvements were also observed in the mean CAT total score $(P<0.001$; Figure 2). Results for the treated sets were similar and are shown in Figures S2 and S3.

The proportion of patients who had an improvement in CCQ total score beyond the established MCID threshold of 0.41 at Month 3 was $71.0 \%$ in DINO and 52.8\% in DACOTA; this increased to $83.3 \%$ and $65.3 \%$ of patients, respectively, at Month 6 (Figure 3A). Similarly, the improvements in CAT total score exceeded the suggested MCID of 2 points in the majority of patients at Month 6 (85.8\% in DINO; $72.8 \%$ in DACOTA; Figure 3B).

In both DINO and DACOTA, all three CCQ domain scores significantly improved from baseline at Months 3 and 6 (all $P<0.001$; data not shown); the greatest mean
Table 2 Patient demographics and baseline characteristics (treated sets)

\begin{tabular}{|c|c|c|}
\hline Characteristic & $\begin{array}{l}\text { DINO } \\
(N=5,375)\end{array}$ & $\begin{array}{l}\text { DACOTA } \\
(\mathbf{N}=3,597)\end{array}$ \\
\hline \multicolumn{2}{|l|}{ (5\%-95\% percentile) (years) } & $67(48-8 I)$ \\
\hline $\begin{array}{l}\text { BMI, median } \\
\text { (5\%-95\% percentile) }\left(\mathrm{kg} / \mathrm{m}^{2}\right)\end{array}$ & $26.8(19.7-36.8)$ & $26.6(19.5-37.3)$ \\
\hline \multicolumn{3}{|l|}{ Sex, n $(\%)^{\mathrm{a}}$} \\
\hline Male & $3,429(63.8)$ & $2,344(65.2)$ \\
\hline Female & $1,943(36.1)$ & $1,249(34.7)$ \\
\hline Unknown & $3(0.1)$ & $4(0.1)$ \\
\hline \multicolumn{3}{|l|}{ Smoking status, $\mathrm{n}(\%)$} \\
\hline Never smoked & $\mathrm{I},|4|(2 \mid .2)$ & $532(14.8)$ \\
\hline Current smoker & $\mathrm{I}, 64 \mathrm{I}(30.5)$ & $963(26.8)$ \\
\hline Former smoker & $2,548(47.4)$ & $2,029(56.4)$ \\
\hline Missing & $45(0.8)$ & $73(2.0)$ \\
\hline \multicolumn{3}{|l|}{ Severity of airway obstruction } \\
\hline \multicolumn{3}{|l|}{ according to GOLD, n (\%) ${ }^{\mathrm{a}, \mathrm{b}}$} \\
\hline Moderate & $195(3.6)$ & $326(9.1)$ \\
\hline Severe & $3,810(70.9)$ & $1,219(33.9)$ \\
\hline Very severe & $1,233(22.9)$ & $267(7.4)$ \\
\hline Unknown/missing & $97(1.8)$ & $\mathrm{I}, 749(48.6)$ \\
\hline \multicolumn{3}{|c|}{ No of moderate or severe exacerbations } \\
\hline \multicolumn{3}{|c|}{ in previous 12 months, $\mathrm{n}(\%)^{\mathrm{a}}$} \\
\hline$<2$ & $2,236(41.6)$ & $\mathrm{I}, 736(48.3)$ \\
\hline$\geq 2$ & $3,092(57.5)$ & $\mathrm{I}, 84 \mid(5 \mid .2)$ \\
\hline Missing & $47(0.9)$ & $20(0.5)$ \\
\hline \multicolumn{3}{|l|}{ Concomitant medications, $\mathrm{n}(\%)^{\mathrm{a}, \mathrm{c}}$} \\
\hline $\begin{array}{l}\text { At least one COPD-specific } \\
\text { maintenance treatment }\end{array}$ & $4,555(84.7)$ & $3,310(92.0)$ \\
\hline SABA & $1,282(23.9)$ & I,775 (49.3) \\
\hline LABA & $\mathrm{I}, 042(19.4)$ & $1,116(31.0)$ \\
\hline SAMA & $130(2.4)$ & $143(4.0)$ \\
\hline LAMA & $2,733(50.8)$ & $2,316(64.4)$ \\
\hline Fixed combination LABA+ICS & $2,390(44.5)$ & I,838 (5I.I) \\
\hline Fixed combination SABA+SAMA & $639(11.9)$ & $695(19.3)$ \\
\hline ICS & $532(9.9)$ & $569(15.8)$ \\
\hline Oral corticosteroid & $759(14.1)$ & $626(17.4)$ \\
\hline Antibiotic & $222(4.1)$ & $192(5.3)$ \\
\hline Combination LABA+LAMA+ICS & $\mathrm{I}, 642(30.5)$ & $1,656(46.0)$ \\
\hline Theophylline & $705(13.1)$ & $636(17.7)$ \\
\hline $\begin{array}{l}\text { Oxygen long-term therapy } \\
(\geq 16 \text { hours/day) }\end{array}$ & $526(9.8)$ & $421(11.7)$ \\
\hline Pulmonary rehabilitation & $106(2.0)$ & $106(2.9)$ \\
\hline \multirow[t]{2}{*}{ Other } & $578(10.8)$ & $213(5.9)$ \\
\hline & $n=4,700$ & $n=3,273$ \\
\hline \multirow[t]{2}{*}{ CCQ total score, mean (SD) } & $3.9(1.0)$ & $3.7(1.0)$ \\
\hline & $n=4,503$ & $n=3,143$ \\
\hline \multirow[t]{2}{*}{ CAT total score, mean (SD) } & $26.8(7.0)$ & $25.4(6.9)$ \\
\hline & & $n=2,173$ \\
\hline $\begin{array}{l}\text { Post-bronchodilator } \mathrm{FEV}_{1} \text {, L, } \\
\text { mean (SD) }\end{array}$ & - & $1.29(0.57)$ \\
\hline
\end{tabular}

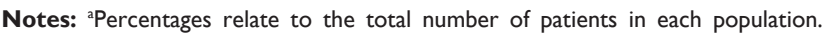
bSeverity of airway obstruction was verified by lung function measurements in DACOTA but not in DINO. 'Multiple answers were possible. $\mathrm{N}=$ total number of patients. $n=$ number of patients with data available.

Abbreviations: BMI, body mass index; CAT, COPD assessment test; CCQ, clinical COPD questionnaire; GOLD, Global Initiative for Chronic Obstructive Lung Disease; ICS, inhaled corticosteroid; LABA, long-acting beta-agonist; LAMA, long-acting muscarinic antagonist; SABA, short-acting beta agonist; SAMA, short-acting muscarinic antagonist. 

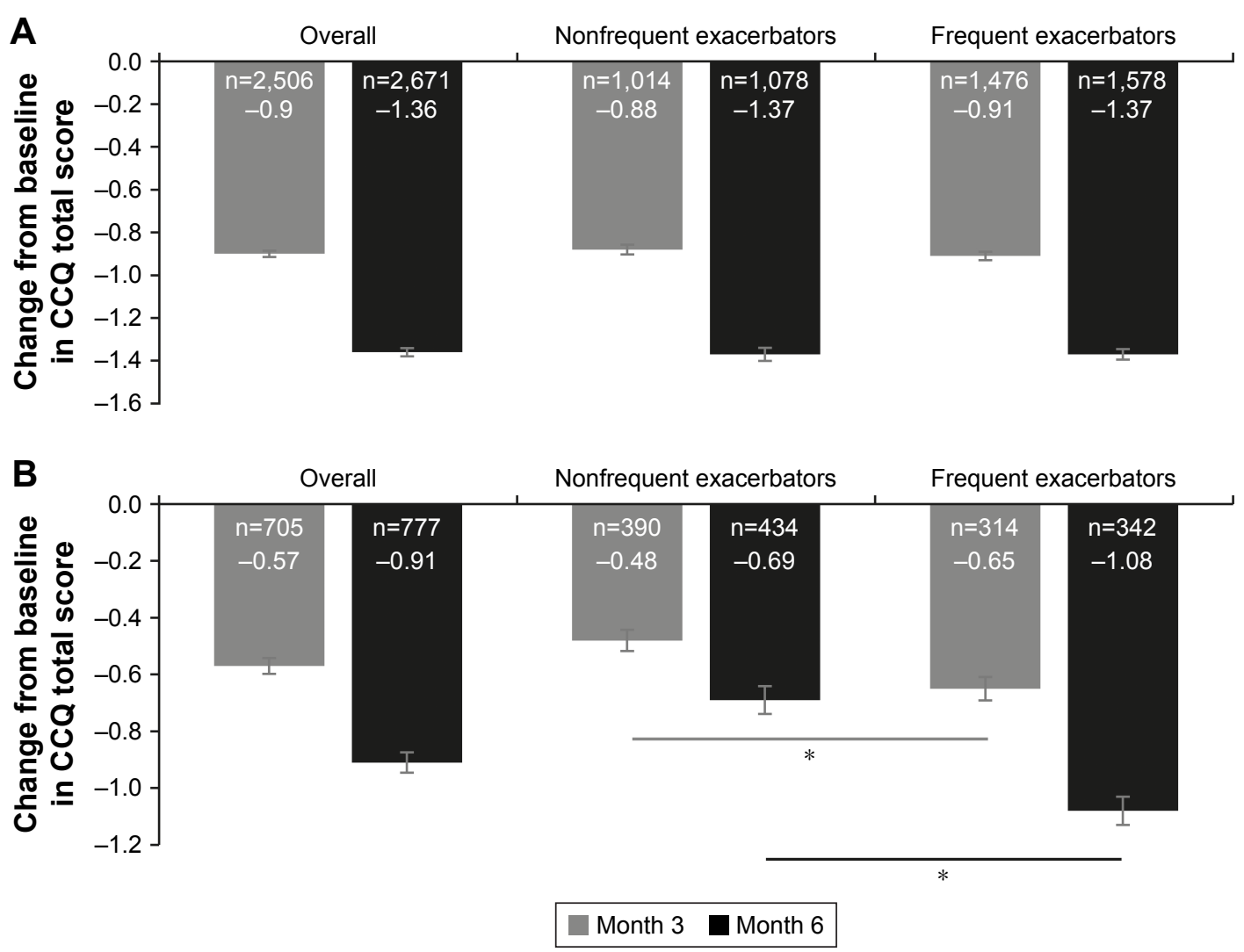

Figure I Change in CCQ total scores from baseline to Months 3 and 6 (effectiveness sets) in the overall population (primary analysis), and in the nonfrequent and frequent exacerbator subgroups (post-hoc analysis) in (A) DINO and (B) DACOTA.

Notes: Data are mean \pm standard error. $n=$ number of patients with data available. All $P<0.00$ I for the changes in $C C Q$ total score from baseline to Months 3 and 6 . $* P<0.00$ I for exacerbation group comparisons.

Abbreviation: CCQ, clinical COPD questionnaire.

change from baseline was in symptom score at Month 6 (DINO: -1.49; DACOTA: -1.07).

\section{Frequent exacerbators}

In both studies, the decreases in CCQ total score and all three domain scores from baseline were statistically significant at Month 6 in both frequent and nonfrequent exacerbator subgroups $(P<0.001$ for CCQ total score, Figure 1; $P<0.001$ for domain scores, data not shown). In DACOTA, the mean improvements in CCQ total score and all domain scores were significantly larger in frequent exacerbators than in nonfrequent exacerbators at both time points $(P<0.001$ for CCQ total score, Figure 1B; $P<0.001$ for CCQ domain scores, data not shown). In DINO, no significant differences were observed between these two subgroups. Similar results were observed in the treated sets (Figure S2).

In DACOTA, mean improvements in CAT total score at both time points were significantly greater in frequent exacerbators compared with nonfrequent exacerbators (Month 3, $P=0.007$; Month 6, $P<0.001$; Figure 2B). However, there were no differences between frequent and nonfrequent exacerbators in DINO. Similar results were observed in the treated sets (Figure S3).

\section{Spirometry}

In DACOTA, post-bronchodilator $\mathrm{FEV}_{1}$, as well as other spirometric variables, significantly improved at both Months 3 and 6 . In the effectiveness set, post-bronchodilator values were available for $\sim 90 \%-100 \%$ of patients at baseline and $40 \%-50 \%$ of patients at Months 3 and 6 . The overall mean change from baseline in $\mathrm{FEV}_{1}$ was $138 \mathrm{~mL}$ at Month $3(P<0.001, \mathrm{n}=396)$ and $202 \mathrm{~mL}$ at Month 6 $(P<0.001, \mathrm{n}=396)$.

\section{Safety}

ADRs were reported by $546(10.2 \%)$ patients in DINO and $661(18.4 \%)$ patients in DACOTA; serious ADRs were reported by $1.1 \%$ of patients in both studies. A total of $413(7.7 \%)$ patients in DINO and 525 (14.6\%) patients in DACOTA discontinued treatment due to an ADR (Table 3). A total of 1,246 ADRs occurring in $500(9.3 \%)$ patients in 

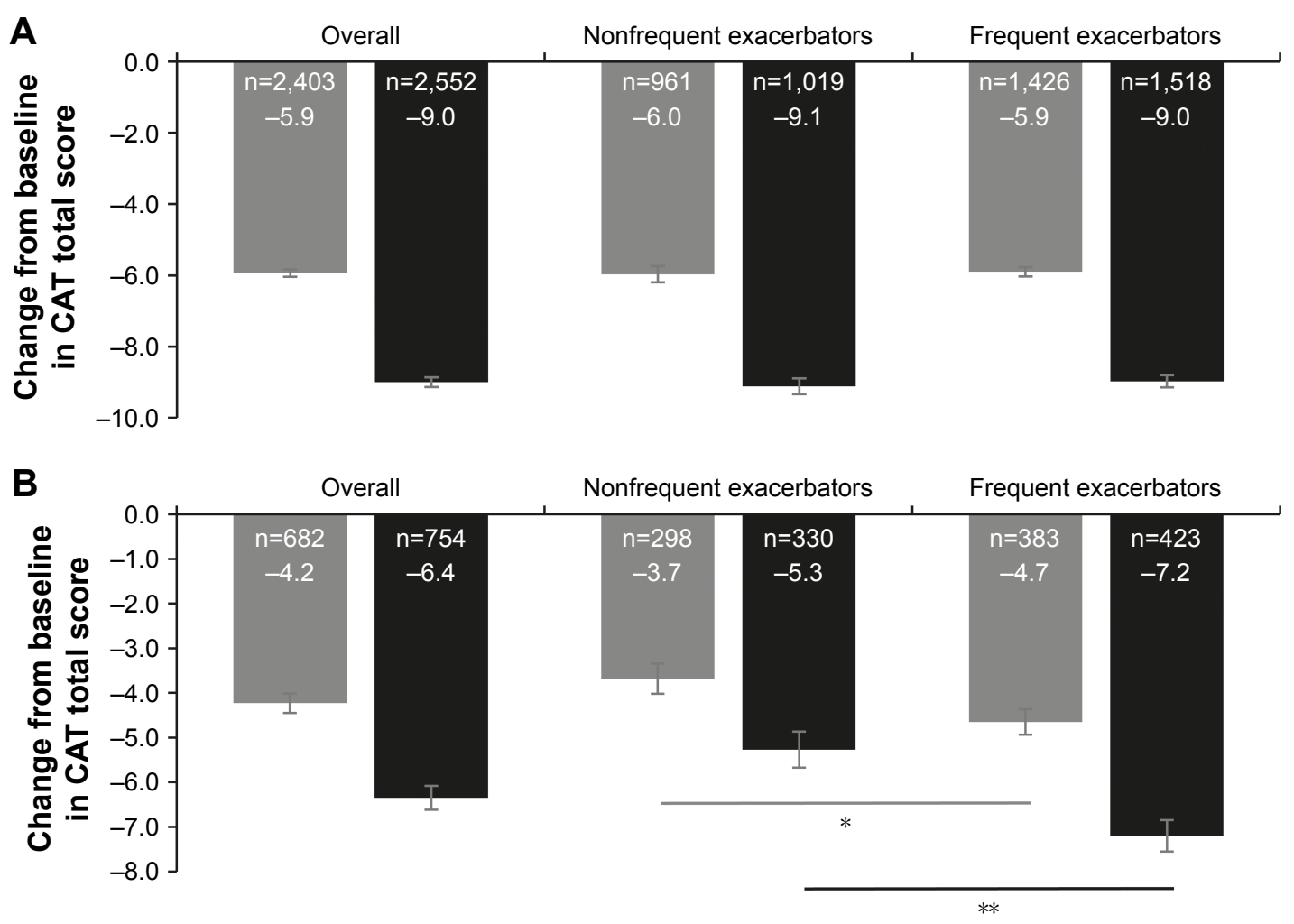

Month 3 Month 6

Figure 2 Change in CAT total scores from baseline to Months 3 and 6 (effectiveness sets) in the overall population (primary analysis), and in the nonfrequent and frequent exacerbator subgroups (post-hoc analysis) in (A) DINO and (B) DACOTA.

Notes: Data are mean \pm standard error: All $P<0.00 \mathrm{I}$ for the changes in CAT total score from baseline to Months 3 and 6 . $* P=0.007$ and $* * P<0.00 \mathrm{I}$ for exacerbation group comparisons.

Abbreviation: CAT, COPD assessment test.

DINO and 1,495 ADRs occurring in 632 (17.6\%) patients in DACOTA were considered causally related to roflumilast. Diarrhea, nausea, and weight decrease were the most frequently reported roflumilast-related ADRs in both studies (Table 3).

\section{Discussion}

The DINO and DACOTA studies included $\sim 9,000$ patients from German primary and secondary specialist respiratory care centers. In both studies, add-on treatment with roflumilast was associated with a significant and clinically relevant improvement in patients' health status, as shown by reductions from baseline in the CCQ total and domain scores, by a level greater than the MCID. These reductions in CCQ total score are in keeping with those shown by Tzanakis et al, who reported a 1.53 point reduction in CCQ score following 6 months of roflumilast treatment in a large real-life Greek COPD cohort. ${ }^{17}$ In DINO and DACOTA, relevant improvements in health status and symptoms were also confirmed by changes in CAT scores. In both studies, improvements in CAT total score from baseline to Month 6 indicate that the average clinical impact of COPD was reduced from a severe (score: $21-30$ ) to a moderate (score: 11-20) impairment.

The improvements in QoL measures in DINO and DACOTA complement the recent findings from DACCORD, another large, noninterventional, real-life study of COPD management in Germany, designed to assess the impact of disease and treatments over 2 years. ${ }^{18,19}$ However, in the most recent roflumilast RCTs REACT and RE'SPOND, health-related QoL, as assessed by the CAT questionnaire, did not improve following addition of roflumilast to existing COPD therapies. ${ }^{4,5}$ An earlier roflumilast RCT (RECORD), reported a trend towards an improvement in health-related QoL, as assessed by the St George's Respiratory Questionnaire. However, the improvement compared with placebo was not significant. ${ }^{20}$ The roflumilast RCTs were not specifically designed to measure QoL and recruited a diverse spectrum of international patients with a range of mind-sets and attitudes, whereas DINO, DACOTA, and DACCORD 

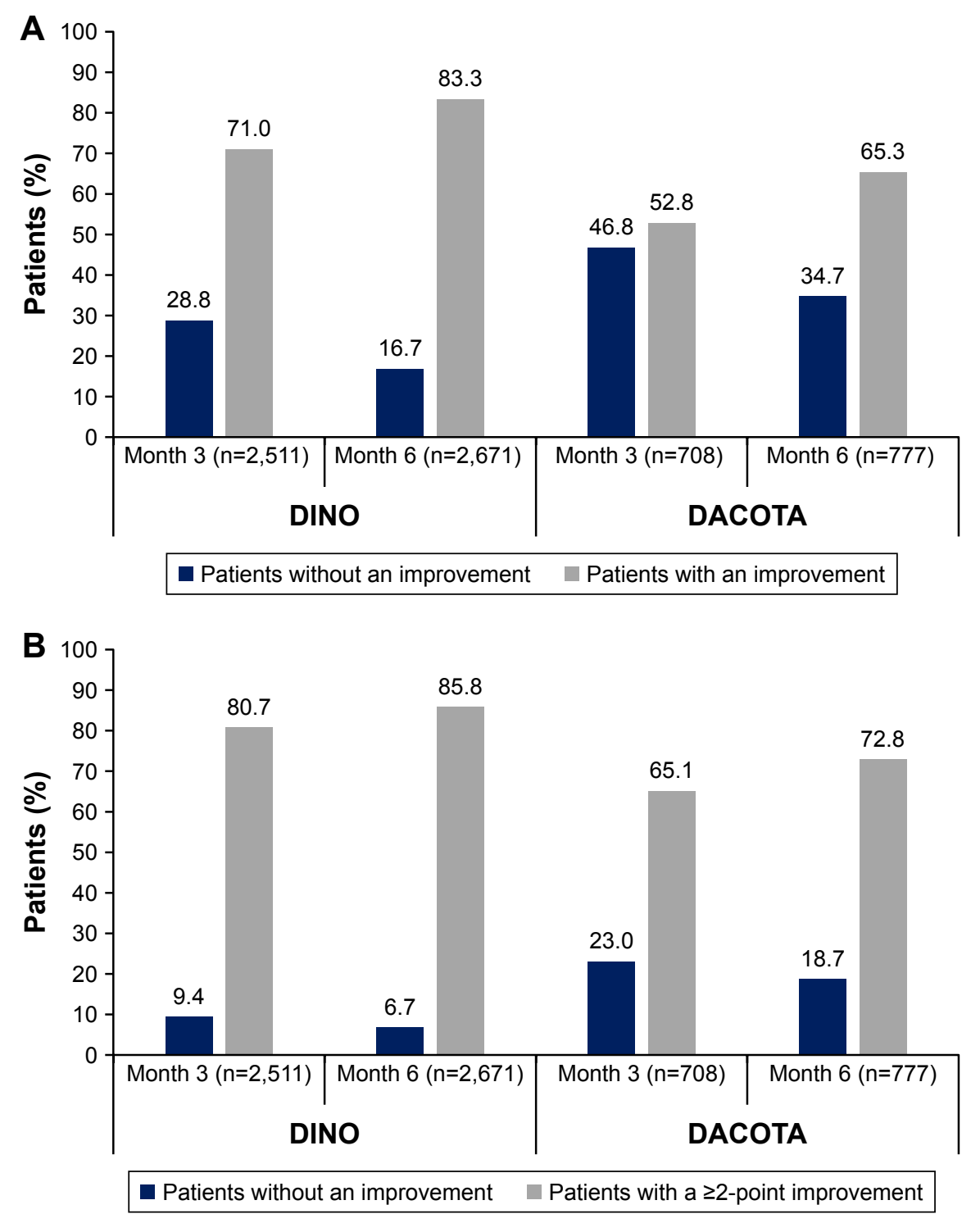

Figure 3 Proportion of patients with a clinically relevant improvement in (A) CCQ total score and (B) CAT score.

Notes: Improvements: change in CCQ total score from baseline $\geq 0.4 \mathrm{I}$ and change in CAT score from baseline $\geq 2$; percentages relate to the total number of patients at each time point.

Abbreviations: CAT, COPD assessment test; CCQ, clinical COPD questionnaire.

were all uncontrolled observational studies and recruited only German patients. This may, at least partially, explain the difference in QoL findings between roflumilast RCTs, and DINO and DACOTA.

In DACOTA, significantly greater improvements in CCQ and CAT scores were observed in patients with a history of frequent moderate or severe exacerbations ( $\geq 2$ in the 12 months prior to roflumilast add-on therapy) than in nonfrequent exacerbators (post-hoc analysis). These data suggest that frequent exacerbators benefited from roflumilast treatment to a larger extent than nonfrequent exacerbators. They also complement the findings from REACT and $\mathrm{RE}^{2} \mathrm{SPOND}$, in which roflumilast improved lung function and reduced exacerbations in patients who had severe disease and frequent exacerbations, despite dual or triple inhaled therapy. ${ }^{4,5}$ It is not clear why greater improvements with roflumilast were also not observed in patients with a history of frequent exacerbations in DINO. In both studies, severity of airflow obstruction was classified by the investigators at baseline according to the GOLD 2011 criteria using data derived from patients' medical records. In DACOTA, a more thorough assessment was conducted with lung function measurements also being taken at baseline in order to verify the severity of obstruction. It is reasonable to assume that patients being treated in secondary care had more severe disease than those treated in primary care, and were therefore 
Table 3 All ADRs and roflumilast-related ADRs occurring in at least $1 \%$ of patients (treated sets)

\begin{tabular}{|c|c|c|}
\hline $\begin{array}{l}\text { ADR occurring in } \geq 1.0 \% \\
\text { of patients by MedDRA } \\
\text { preferred term, } n(\%)^{\mathrm{a}}\end{array}$ & $\begin{array}{l}\text { DINO } \\
(N=5,375)\end{array}$ & $\begin{array}{l}\text { DACOTA } \\
(\mathbf{N}=3,597)\end{array}$ \\
\hline All ADRs & $546(10.2)$ & $661(18.4)$ \\
\hline $\begin{array}{l}\text { ADR leading to } \\
\text { premature discontinuation }\end{array}$ & $4 \mid 3(7.7)$ & $525(14.6)$ \\
\hline Nausea & $197(3.7)$ & $203(5.6)$ \\
\hline Diarrhea & $186(3.5)$ & $279(7.8)$ \\
\hline Weight decrease ${ }^{b}$ & $68(1.3)$ & $95(2.6)$ \\
\hline Headache & - & $65(1.8)$ \\
\hline Dizziness & - & $65(1.8)$ \\
\hline Decreased appetite & - & $45(1.3)$ \\
\hline Vomiting & - & $41(1.1)$ \\
\hline Sleep disorder ${ }^{c}$ & - & $54(1.5)$ \\
\hline Abdominal pain & - & $38(I . I)$ \\
\hline Tremor & - & $36(1.0)$ \\
\hline Roflumilast-related $A D R s^{d}$ & $500(9.3)$ & $632(17.6)$ \\
\hline Diarrhea & $176(3.3)$ & $276(7.7)$ \\
\hline Nausea & $190(3.5)$ & $199(5.5)$ \\
\hline Weight loss ${ }^{\mathrm{b}}$ & $63(1.2)$ & $94(2.6)$ \\
\hline Headache & - & $63(1.8)$ \\
\hline Decreased appetite & - & $45(1.3)$ \\
\hline Vomiting & - & $4 I(1.1)$ \\
\hline Sleep disorder ${ }^{c}$ & - & $52(1.4)$ \\
\hline Abdominal pain & - & $38(1.1)$ \\
\hline Tremor & - & $35(1.0)$ \\
\hline
\end{tabular}

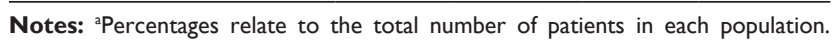
'In addition, one patient in DINO (all ADRs and roflumilast-related ADRs) was reported with the preferred term "abnormal loss of weight". 'In addition, I 4 patients in DINO and 30 patients in DACOTA (all ADRs and roflumilast-related ADRs)

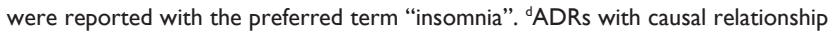
to roflumilast as assessed by the investigators or ADRs with unknown/missing relationship to roflumilast.

Abbreviations: ADR, adverse drug reaction; MedDRA, Medical Dictionary for Regulatory Activities.

likely to experience greater improvements with roflumilast. We also speculate that specialists manage exacerbations more commonly than their primary care counterparts, and may be able to identify and record these events more accurately. Better recording of exacerbations in the secondary care setting, compared with that in the primary care setting, may have allowed us to distinguish frequent from nonfrequent exacerbators more accurately.

Significant improvements in lung function were observed during the course of DACOTA, with the improvement in post-bronchodilator $\mathrm{FEV}_{1}$ far greater than has been reported in RCTs $(202 \mathrm{~mL}$ over 6 months in DACOTA versus $\sim 50 \mathrm{~mL}$ over 1 year in RCTs)., ${ }^{2,40}$ The reasons for this finding are not fully understood, but results may have been influenced by the healthy survivor effect - a potential source of bias that is discussed in more detail below. This finding may also partially be attributed to the real-life nature of DACOTA.
It is well known that adherence to inhaled medication can be poor, especially in patients with COPD, and that many patients use their inhalers incorrectly. While roflumilast is not a direct bronchodilator, $\mathrm{FEV}_{1}$ improvements could have been precipitated by the general anti-inflammatory effects of starting this systemic therapy, which translated into improved lung function..$^{21}$ Additionally, spirometry in DACOTA was random, missing for $48.6 \%$ of patients in the treated set at baseline, and there were no restrictions regarding the time point of spirometry or the time point of roflumilast intake. It is important to note that $\mathrm{FEV}_{1}$ was not a primary endpoint in DACOTA, and that all the participating centers were secondary care respiratory clinics. Therefore, unlike many registry studies and DINO, patients included in DACOTA were very likely to have fulfilled the criteria for a diagnosis of COPD. Furthermore, in real-life noninterventional studies, the use of spirometry is at the discretion of the treating physician. However, the frequency of spirometry observed in DACOTA is in line with that from previous studies, which have reported an absence of spirometry in $\sim 40 \%-60 \%$ of COPD cases in everyday clinical practice. ${ }^{22-24}$ Nevertheless, all of these factors may have influenced the measurements, and caution should be taken when interpreting these data.

The tolerability profile of roflumilast was consistent with that determined in clinical studies, with no new safety concerns arising. The proportion of patients who discontinued treatment was $33.8 \%$ in DACOTA and $30.6 \%$ in DINO, in line with recent RCTs, in which $28 \%$ discontinued in REACT and 29\% discontinued in $\mathrm{RE}^{2} \mathrm{SPOND},{ }^{4,5}$ and a search of clinical databases (December 2012-April 2015) in Korea, which reported a discontinuation rate of $35.1 \%{ }^{25}$ The proportion of patients who discontinued treatment due to an ADR was $7.7 \%$ in DINO and $14.6 \%$ in DACOTA. Although these rates are within the range of those reported in RCTs, 4,5 the difference between DINO and DACOTA could reflect the more frequent patient-physician contact in the primary care setting than in the secondary care setting.

ADRs associated with roflumilast, for example, diarrhea and weight loss, commonly reported during the first few months of therapy, are typical of many systemic therapies and may not be a barrier to initiating therapy. ${ }^{3}$ The finding that measures of QoL improved during the 6 months of roflumilast treatment suggests that patients on roflumilast treatment may not have been overly affected by ADRs. A number of studies using alternative dosing strategies have been conducted with the aim of improving patients' adherence. For example, a small-scale retrospective study and a number of modeling studies have indicated that initiating a reduced dose of $250 \mu \mathrm{g}$ 
for the first 4 weeks of therapy before uptitrating to the approved dose may help improve adherence to roflumilast for those patients who are affected by ADRs. ${ }^{26-28}$ Results from the OPTIMIZE study have confirmed this by demonstrating a $34 \%$ reduction in the odds of discontinuing therapy when an initial dose of roflumilast $250 \mu \mathrm{g}$ OD is taken for 4 weeks before uptitration to $500 \mu \mathrm{g}$ OD. ${ }^{29}$ These strategies were not in effect at the time of DINO and DACOTA.

Overall, $4.3 \%$ of patients in DINO and $10.1 \%$ of patients in DACOTA had mild or moderate disease as assessed by GOLD 2011 and were therefore treated off-label. These patients were not excluded from the treated set data analyses due to the noninterventional nature of the study. The majority of patients in both studies received at least one COPDspecific maintenance treatment in addition to roflumilast. However, patterns of medication use in real life do not always reflect that of RCTs and the label. For example, ICS was taken as a monotherapy in $9.9 \%$ of patients in DINO and $15.8 \%$ in DACOTA, despite not being indicated for use as a monotherapy in COPD. It is possible that these patients could have been using an additional single inhaler, such as a LABA or LAMA, or were receiving ICS monotherapy as a result of an earlier diagnosis of asthma in primary care. Subgroup analyses according to concomitant treatment were beyond the scope of these studies; however, further investigations on the effectiveness of roflumilast with different combinations of concomitant therapies could be informative. Consequently, results on the effectiveness of roflumilast should be interpreted with some caution, given this lack of information on previously prescribed medications. Furthermore, results from the DINO and DACOTA studies were considered in the context of the GOLD 2011 Guidelines, which were subsequently updated in 2018, and recommended the use of roflumilast as maintenance treatment (added-on to inhaled therapy) only in patients with $\mathrm{FEV}_{1}<50 \%$ (predicted) associated with chronic bronchitis and a history of exacerbations.

DINO and DACOTA were prospective noninterventional studies designed to assess clinical treatment effects and tolerability, including rare ADRs, in a large and heterogeneous group of individuals under conditions of everyday clinical practice. The limitations of these studies are those associated with their nonexperimental design and include high drop-out rates, missing data, and the lack of a control or comparator with regard to the evaluation of treatment effects. However, the inclusion of such a comparator in these nonrandomized observational studies may have introduced selection biases. Other limitations include the inclusion of patients with at least one dose of roflumilast, the effects of potential confounding by indication bias, where the most severe patients may preferentially receive certain treatments, ${ }^{7}$ and a lack of generalizability due to the studies being conducted solely in a German population. Nevertheless, although DINO and DACOTA were carried out in a German population, these findings may be generalized to patients with COPD in other European Union countries with comparable reimbursement systems. Additionally, the lack of placebo means any imbalance of patient- and investigator-related effects cannot be accounted for. ${ }^{7}$ Another important source of bias in real-life studies is that of attrition. ${ }^{7}$ For example, in DACCORD, almost half of the 6,000 patients initially included in the study failed to complete the 2-year visit. ${ }^{18,30}$ A high proportion of patients ( $40 \%$ of the enrolled population in DINO and $75 \%$ of the enrolled population in DACOTA) were excluded from the effectiveness set analyses, which means we should exercise caution when extrapolating these data. An important factor to consider when attrition rates are high is the healthy survivor effect - that is, patients who perceive a benefit with treatment will persevere with treatment more than patients who do not perceive a benefit. However, in both studies, baseline characteristics and endpoint data were similar for both the treated and effectiveness analysis sets, suggesting that the impact of attrition on these studies was minor.

Despite the known limitations of noninterventional studies, the results of these two independent observational studies are encouraging and demonstrate that roflumilast add-on therapy improves QoL in patients with COPD in a real-life clinical setting. These studies also reveal interesting trends in the use of roflumilast in clinical practice, which warrant further investigation.

\section{Acknowledgments}

DINO and DACOTA were sponsored by Takeda; AstraZeneca is the current study sponsor. Rebecca Birch, $\mathrm{PhD}$, provided medical writing support, under guidance from the authors, on behalf of Synergy Vision, London, UK, funded by AstraZeneca, UK, in accordance with Good Publication Practice (GPP3) guidelines (http://www.ismpp.org/gpp3). Kushal Banerjee, $\mathrm{PhD}$, provided medical writing support in addressing the peer reviewer comments, under guidance from the authors, on behalf of Cactus Communications, Mumbai, India, funded by AstraZeneca, UK, in accordance with GPP3 guidelines.

\section{Disclosure}

IM is a former employee of Takeda and a current employee of AstraZeneca. PK reports personal fees from Takeda during the 
conduct of the study; personal fees from AstraZeneca, Chiesi, GSK, Novartis, Menarini, Teva, and Mundipharma outside the submitted work. CV reports no relevant conflicts of interest in the current study, and the following relevant financial activities outside of the submitted work: personal fees from Almirall, Cipla, Berlin Chemie/Menarini, CSL Behring, and Teva; grants and personal fees from AstraZeneca, Boehringer Ingelheim, Chiesi, GlaxoSmithKline, Grifols, Mundipharma, Novartis, and Takeda; grants from German Federal Ministry of Education and Research (BMBF) Competence Network Asthma and COPD (ASCONET), Bayer Schering Pharma AG, MSD, and Pfizer. RS reports no conflicts of interest in this work. The authors report no other conflicts of interest in this work.

\section{References}

1. Global Initiative for Chronic Obstructive Lung Disease (GOLD) [homepage on the Internet]. Global Strategy for the Diagnosis, Management, and Prevention of COPD, 2018. Available from: http:// goldcopd.org/. Accessed December 9, 2017.

2. Calverley PM, Rabe KF, Goehring UM, Kristiansen S, Fabbri LM, Martinez FJ. Roflumilast in symptomatic chronic obstructive pulmonary disease: two randomised clinical trials. Lancet. 2009;374(9691): 685-694.

3. Fabbri LM, Calverley PM, Izquierdo-Alonso JL, et al. Roflumilast in moderate-to-severe chronic obstructive pulmonary disease treated with longacting bronchodilators: two randomised clinical trials. Lancet. 2009;374(9691):695-703.

4. Martinez FJ, Calverley PM, Goehring UM, Brose M, Fabbri LM, Rabe KF. Effect of roflumilast on exacerbations in patients with severe chronic obstructive pulmonary disease uncontrolled by combination therapy (REACT): a multicentre randomised controlled trial. Lancet. 2015;385(9971):857-866.

5. Martinez FJ, Rabe KF, Sethi S, et al. Effect of roflumilast and inhaled corticosteroid/long-acting beta2-agonist on chronic obstructive pulmonary disease exacerbations (RE(2)SPOND). A randomized clinical trial. Am J Respir Crit Care Med. 2016;194(5):559-567.

6. Vos W, Hajian B, De Backer J, et al. Functional respiratory imaging to assess the interaction between systemic roflumilast and inhaled ICS/ LABA/LAMA. Int J Chron Obstruct Pulmon Dis. 2016;11:263-271.

7. Kardos P, Worsley S, Singh D, Roman-Rodriguez M, Newby DE, Mullerova H. Randomized controlled trials and real-world observational studies in evaluating cardiovascular safety of inhaled bronchodilator therapy in COPD. Int J Chron Obstruct Pulmon Dis. 2016;11: 2885-2895.

8. Herland K, Akselsen JP, Skjonsberg OH, Bjermer L. How representative are clinical study patients with asthma or COPD for a larger "real life" population of patients with obstructive lung disease? Respir Med. 2005;99(1):11-19.

9. Chong J, Leung B, Poole P. Phosphodiesterase 4 inhibitors for chronic obstructive pulmonary disease. Cochrane Database Syst Rev. 2013; (11):CD002309.

10. Summary of Product Characteristics (Europe): Daxas ${ }^{\circledR} 500$ microgram film-coated tablets [webpage on the Internet]. AstraZeneca Pharmaceuticals, 2016. Available from: http://www.ema.europa.eu/ema/. Accessed December 9, 2017.

11. World Medical Association Declaration of Helsinki: ethical principles for medical research involving human subjects. $J$ Int Bioethique. 2004;15(1):124-129.
12. Epstein M. Guidelines for good pharmacoepidemiology practices (GPP). Pharmacoepidemiol Drug Saf. 2005;14(8):589-595.

13. van der Molen T, Willemse BW, Schokker S, ten Hacken NH, Postma DS, Juniper EF. Development, validity and responsiveness of the Clinical COPD Questionnaire. Health Qual Life Outcomes. 2003;1:13.

14. Jones PW, Harding G, Berry P, Wiklund I, Chen WH, Kline Leidy N. Development and first validation of the COPD Assessment Test. Eur Respir J. 2009;34(3):648-654.

15. Kocks JW, Tuinenga MG, Uil SM, van den Berg JW, Stahl E, van der Molen T. Health status measurement in COPD: the minimal clinically important difference of the clinical COPD questionnaire. Respir Res. 2006;7:62.

16. Kon SSC, Canavan JL, Jones SE, et al. Minimum clinically important difference for the COPD Assessment Test: a prospective analysis. Lancet Respir Med. 2014;2(3):195-203.

17. Tzanakis N, Ginis A, Politis G, et al. Quality of life and symptoms improvement after 6 months treatment with roflumilast $500 \mu \mathrm{g}$ daily in a large Greek COPD cohort. Eur Respir J. 2013;42(Suppl 57):P2385.

18. Buhl R, Criee CP, Kardos P, et al. A year in the life of German patients with COPD: the DACCORD observational study. Int J Chron Obstruct Pulmon Dis. 2016;11:1639-1646.

19. Kardos P, Vogelmeier C, Worth H, et al. A two-year evaluation of the 'real life' impact of COPD on patients in Germany: the DACCORD observational study. Respir Med. 2017;124:57-64.

20. Rabe KF, Bateman ED, O'Donnell D, Witte S, Bredenbroker D, Bethke TD. Roflumilast - an oral anti-inflammatory treatment for chronic obstructive pulmonary disease: a randomised controlled trial. Lancet. 2005;366(9485):563-571.

21. De Backer W, Vos W, Van Holsbeke C, et al. The effect of roflumilast in addition to LABA/LAMA/ICS treatment in COPD patients. Eur Respir J. 2014;44:527-529.

22. Han MK, Kim MG, Mardon R, et al. Spirometry utilization for COPD: how do we measure up? Chest. 2007;132(2):403-409.

23. Miravitlles M, de la Roza C, Naberan K, Lamban M, Gobartt E, Martin A. Use of spirometry and patterns of prescribing in COPD in primary care. Respir Med. 2007;101(8):1753-1760.

24. Arne M, Lisspers K, Ställberg B, et al. How often is diagnosis of COPD confirmed with spirometry? Respir Med. 2010;104(4):550.

25. Kim KH, Kang HS, Kim JS, Yoon HK, Kim SK, Rhee CK. Risk factors for the discontinuation of roflumilast in patients with chronic obstructive pulmonary disease. Int J Chron Obstruct Pulmon Dis. 2017;12: 3449-3456.

26. Lahu G, Hunnemeyer A, Diletti E, et al. Population pharmacokinetic modelling of roflumilast and roflumilast $\mathrm{N}$-oxide by total phosphodiesterase-4 inhibitory activity and development of a population pharmacodynamic-adverse event model. Clin Pharmacokinet. 2010; 49(9):589-606.

27. Lahu G, Facius A. Application of population pharmacokinetic modeling to explore the impact of alternative roflumilast dosing regimens on tolerability. Int J Clin Pharmacol Ther. 2013;51(11):832-836.

28. Hwang H, Shin JY, Park KR, et al. Effect of a dose-escalation regimen for improving adherence to roflumilast in patients with chronic obstructive pulmonary disease. Tuberc Respir Dis (Seoul). 2015;78(4): 321-325.

29. Rabe KF, Rennard S, Alagappan VKT, et al. Use of a 4-week uptitration regimen of roflumilast in patients with severe COPD. Int $J$ Chron Obstruct Pulmon Dis. 2018;13:813-822.

30. Worth H, Buhl R, Criee CP, Kardos P, Mailander C, Vogelmeier C. The 'real-life' COPD patient in Germany: the DACCORD study. Respir Med. 2016;111:64-71. 


\section{Supplementary materials Effectiveness set criteria}

In both DINO and DACOTA, the effectiveness set was defined as a population that was more compliant with the label and the study protocol and had complete data available for the assessment of the primary endpoint. The effectiveness set was based on patients in the treated set who met the following criteria:

- Treatment with roflumilast for $\geq 5$ months.

- No simultaneous initiation of any other long-term $(\geq 3$ months) COPD-specific maintenance treatments/therapies; short-acting beta-agonists (SABA) and/or short-acting anticholinergic (SAMA) were allowed.

- Post-bronchodilator $\mathrm{FEV}_{1}<50 \%$ predicted.

- Evaluable assessments of clinical COPD questionnaire at baseline and after 6 months.

- No simultaneous initiation of short-term ( $\leq 14$ days) treatment with oral corticosteroids, antibiotics, or theophylline.

- Start date of roflumilast treatment not $>7$ days prior to Visit 1 (baseline; DACOTA only).

\section{A}

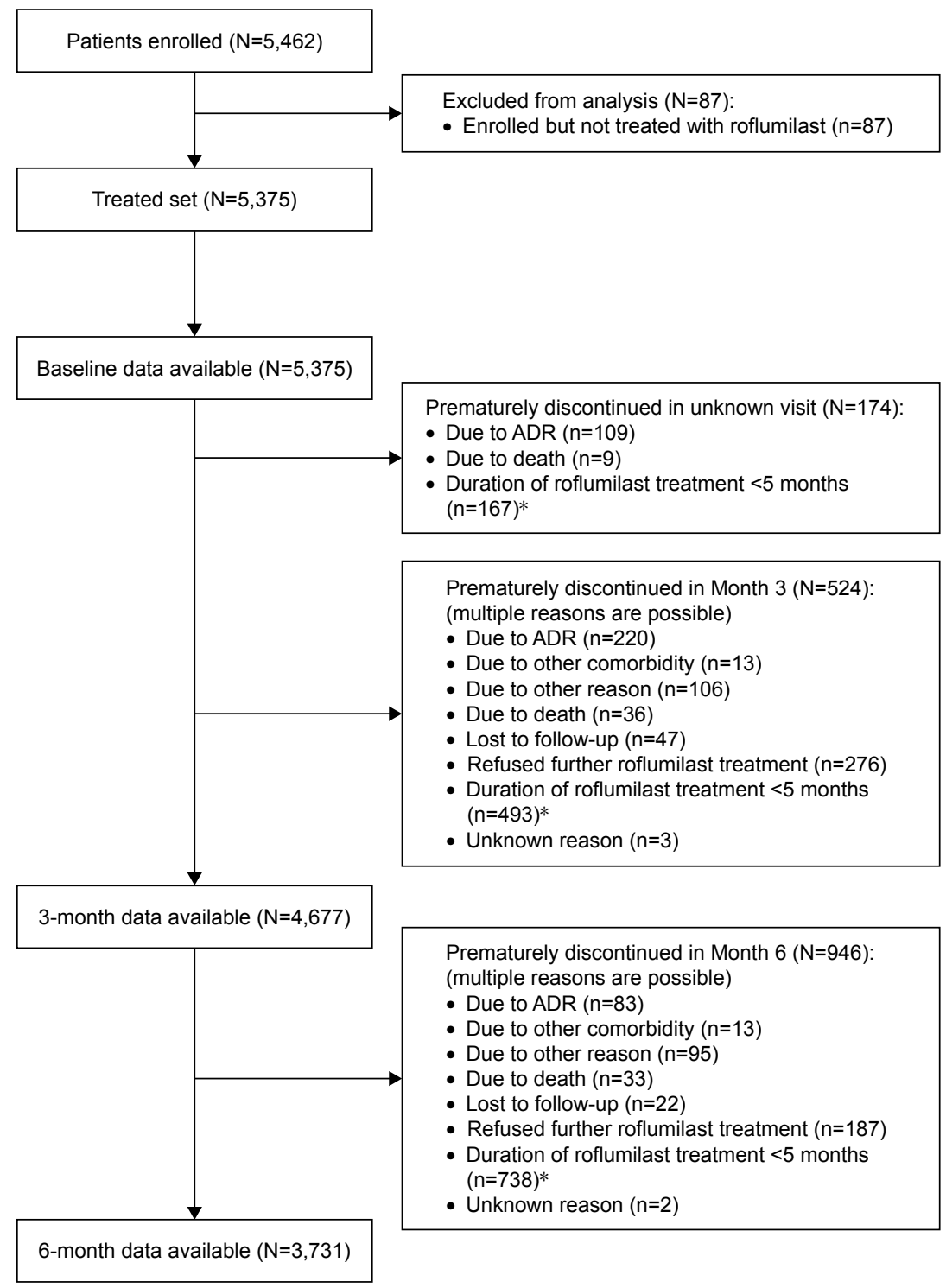

Figure SI (Continued) 
B

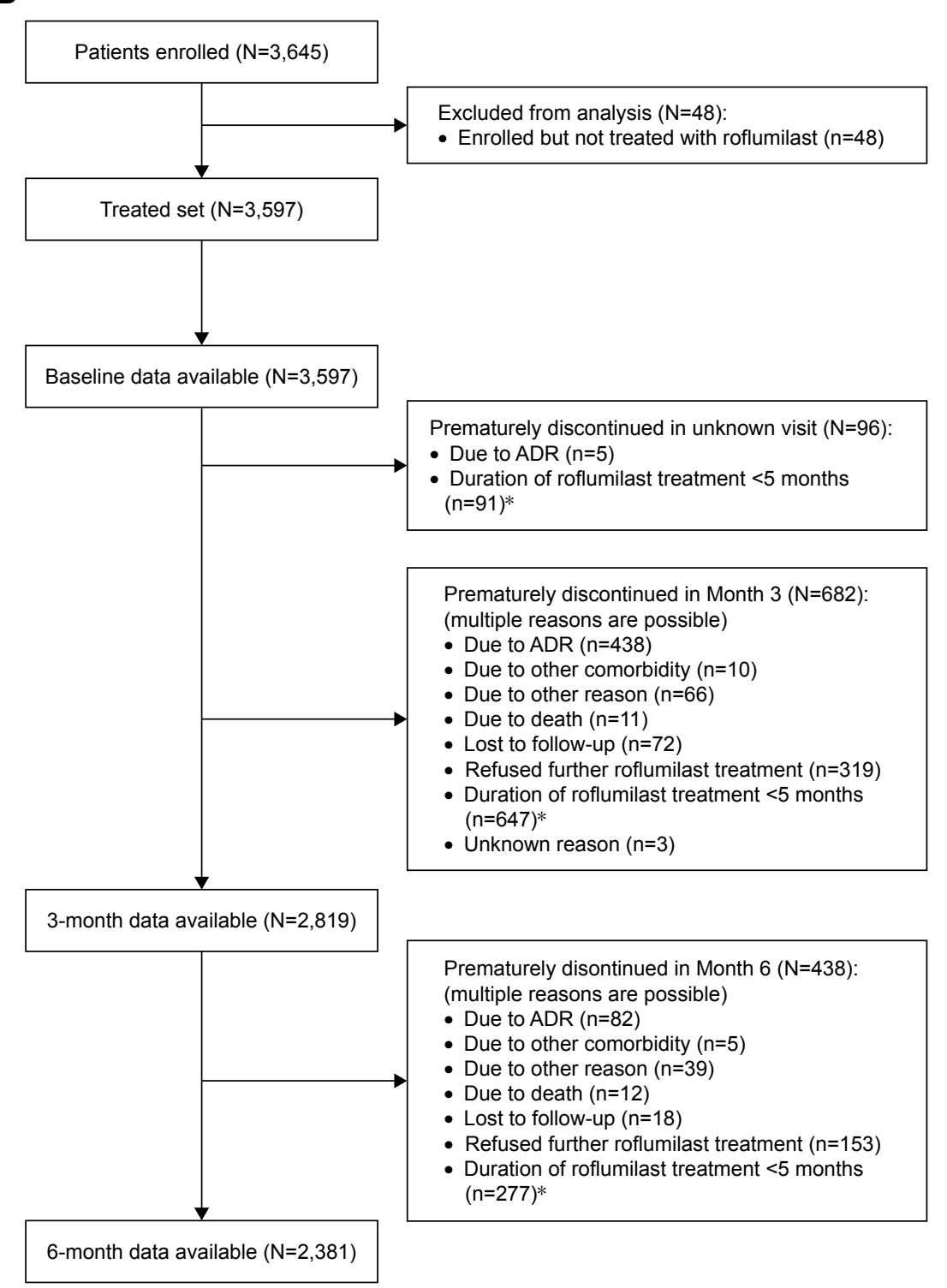

Figure SI Patient disposition (treated sets) in (A) DINO and (B) DACOTA.

Notes: *Duration of roflumilast treatment $<5$ months, as reason for premature discontinuation, was not available as a check box in the CRF but was included for the purpose of the analysis. $\mathrm{N}=$ total number of patients. $\mathrm{n}=$ number of patients with an event.

Abbreviations: $A D R$, adverse drug reaction; CRF, case report form. 
Table SI Patient demographics and baseline characteristics (effectiveness sets)

\begin{tabular}{|c|c|c|}
\hline Characteristic & $\begin{array}{l}\text { DINO } \\
N=3,274\end{array}$ & $\begin{array}{l}\text { DACOTA } \\
\mathrm{N}=916\end{array}$ \\
\hline Age, median (5\%-95\% percentile) (years) & $67(48-82)$ & $66(50-79)$ \\
\hline BMI, median (5\%-95\% percentile) $\left(\mathrm{kg} / \mathrm{m}^{2}\right)$ & $26.8(20.1-36.6)$ & $26.6(19.7-37.7)$ \\
\hline \multicolumn{3}{|l|}{ Sex, n (\%) } \\
\hline Male & $2,100(64.1)$ & $60 \mathrm{I}(65.6)$ \\
\hline Female & I,I7I (35.8) & $314(34.3)$ \\
\hline Unknown & $3(0.1)$ & $\mathrm{I}(0.1)$ \\
\hline \multicolumn{3}{|c|}{ Severity of airway obstruction according to GOLD, $\mathrm{n}(\%)^{\mathrm{a}}$} \\
\hline Mild & 0 & 0 \\
\hline Moderate & 0 & 0 \\
\hline Severe & $2,555(78.0)$ & 731 (79.8) \\
\hline Very severe & $719(22.0)$ & $138(15.1)$ \\
\hline \multirow[t]{2}{*}{ Unknown/missing } & 0 & $47(5.1)$ \\
\hline & $\mathrm{n}=\mathbf{3 , 2 7 4}$ & $n=916$ \\
\hline \multirow[t]{2}{*}{ CCQ total score, mean (SD) } & $3.9(1.0)$ & $3.8(1.0)$ \\
\hline & $n=3,151$ & $n=891$ \\
\hline \multirow[t]{2}{*}{ CAT total score, mean (SD) } & $27.1(6.8)$ & $26.4(6.5)$ \\
\hline & & $\mathrm{n}=906$ \\
\hline Post-bronchodilator $\mathrm{FEV}_{1}$, L, mean (SD) & - & $\mathrm{I} .14(0.47)$ \\
\hline
\end{tabular}

Notes: aPercentages relate to the total number of patients in each population. $\mathrm{N}=$ total number of patients. $\mathrm{n}=$ number of patients with data available.

Abbreviations: BMI, body mass index; CAT, COPD assessment test; CCQ, clinical COPD questionnaire; GOLD, Global Initiative for Chronic Obstructive Lung Disease.
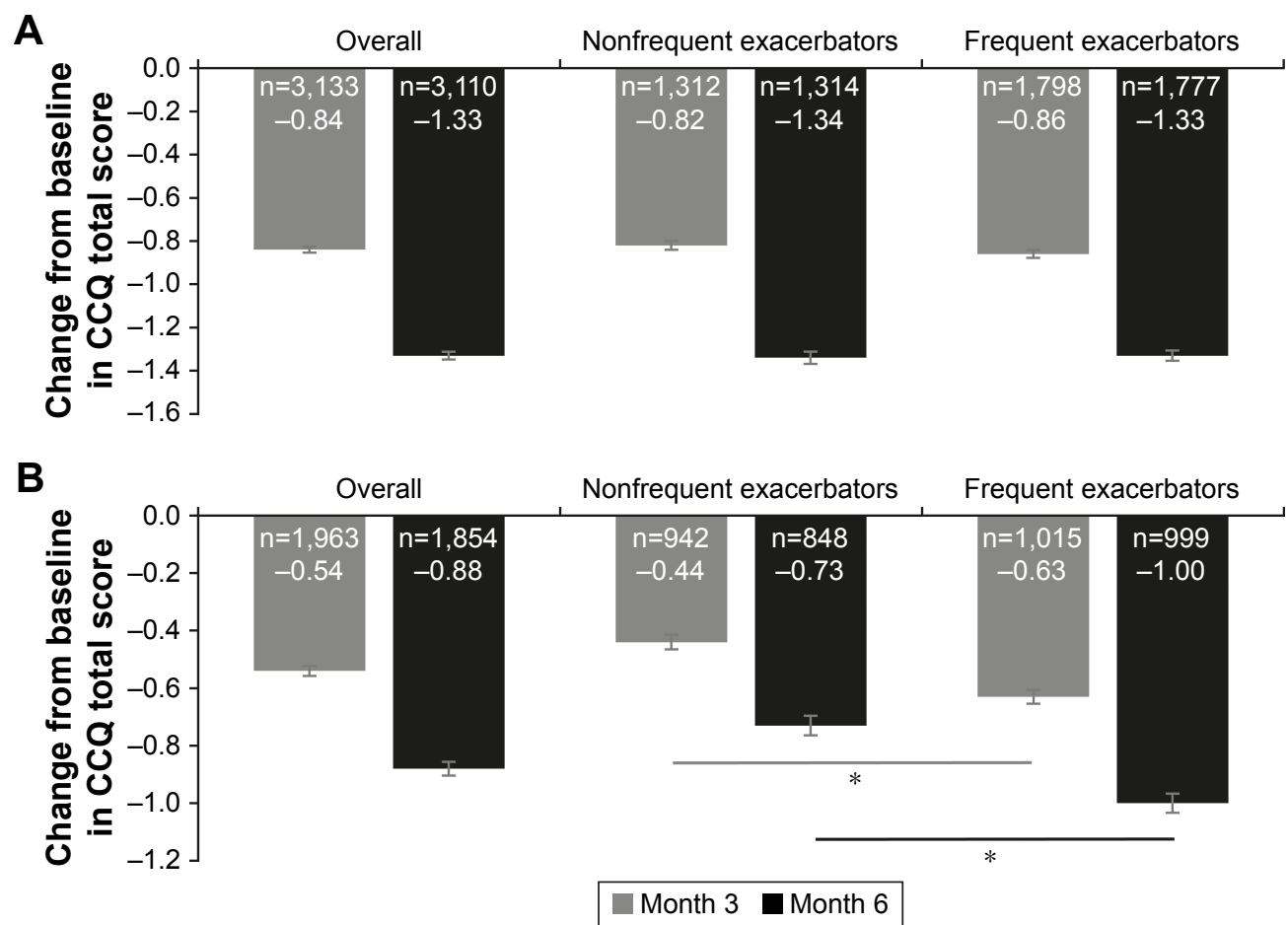

Figure S2 Change in CCQ total scores from baseline to Months 3 and 6 (treated sets) in the overall population (primary analysis), and in the frequent and nonfrequent exacerbator subgroups (post-hoc analysis) in (A) DINO and (B) DACOTA.

Notes: Data are mean \pm standard error. $n=$ number of patients with data available. All $P<0.00$ I for the changes in CCQ total score from baseline to months 3 and 6 . $* P<0.00$ I for exacerbation group comparisons.

Abbreviation: $C C Q$, clinical COPD questionnaire. 

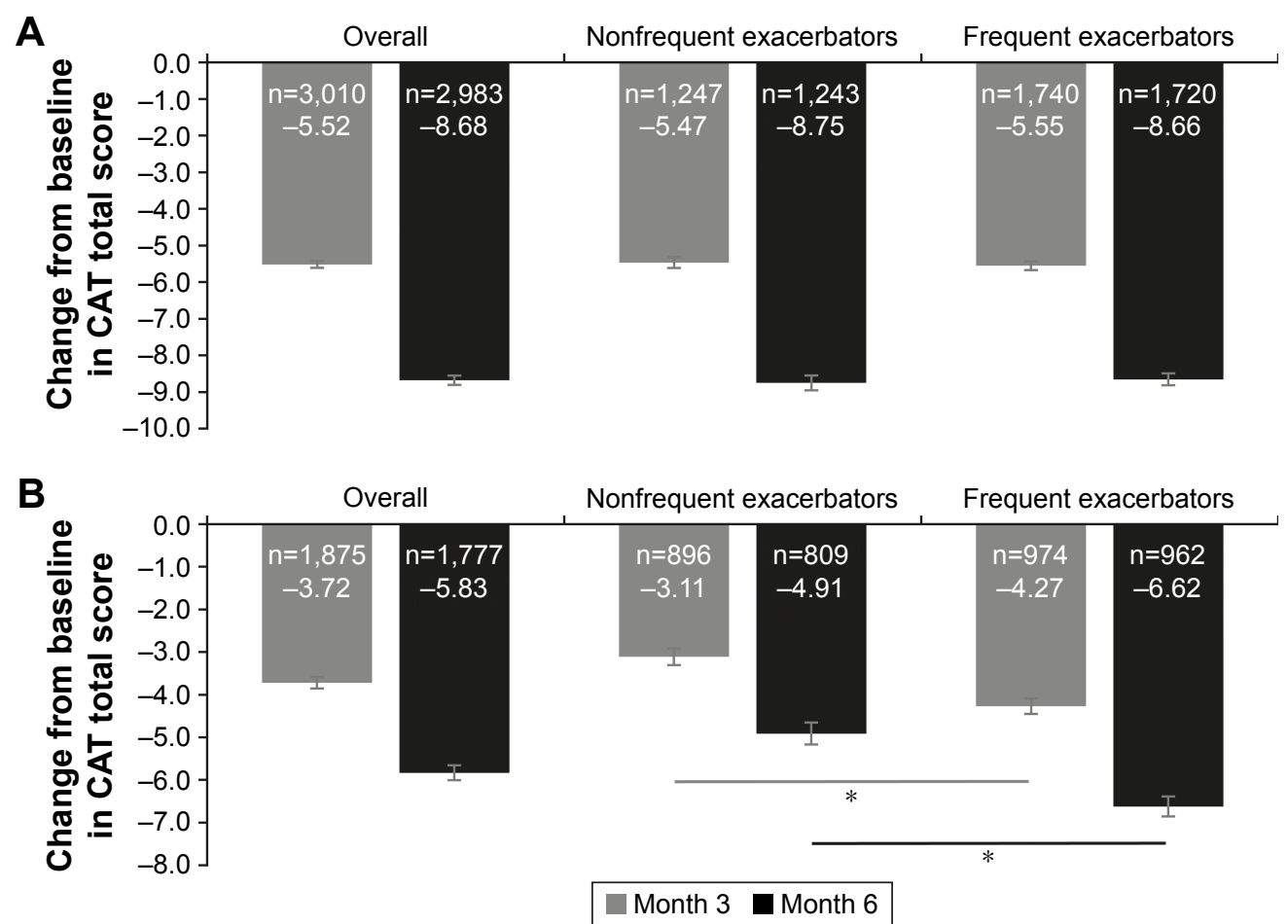

Figure S3 Change in CAT total scores from baseline to months 3 and 6 (treated sets) in the overall population (primary analysis), and in the nonfrequent and frequent exacerbator subgroups (post-hoc analysis) in (A) DINO and (B) DACOTA.

Notes: Data are mean \pm standard error. All $P<0.00$ I for the changes in CAT total score from baseline to Months 3 and 6 . $* P<0.001$ for exacerbation group comparisons. Abbreviation: CAT, COPD assessment test.

\section{Publish your work in this journal}

The International Journal of COPD is an international, peer-reviewed journal of therapeutics and pharmacology focusing on concise rapid reporting of clinical studies and reviews in COPD. Special focus is given to the pathophysiological processes underlying the disease, intervention programs, patient focused education, and self management protocols.
This journal is indexed on PubMed Central, MedLine and CAS. The manuscript management system is completely online and includes a very quick and fair peer-review system, which is all easy to use. Visit http://www.dovepress.com/testimonials.php to read real quotes from published authors. 